\title{
AICAR enhances insulin signaling via downregulation of miR-29
}

\begin{tabular}{|c|c|}
\hline Journal: & Canadian Journal of Physiology and Pharmacology \\
\hline Manuscript ID & cjpp-2015-0159.R1 \\
\hline Manuscript Type: & Article \\
\hline Date Submitted by the Author: & $21-J u l-2015$ \\
\hline Complete List of Authors: & $\begin{array}{l}\text { Liu, Jia; } 2011 \text { Collaborative Innovation Center of Tianjin for Medical } \\
\text { Epigenetics, Key Laboratory of Hormones and Development (Ministry of } \\
\text { Health), Metabolic Diseases Hospital \& Tianjin Institute of Endocrinology, } \\
\text { Tianjin Medical University, } 66 \text { Tong-an Road } \\
\text { Ye, Cheng; Key Laboratory of Food Safety Research, Institute for } \\
\text { Nutritional Sciences, Shanghai Institutes for Biological Sciences, Chinese } \\
\text { Academy of Sciences, } 320 \text { Yue-yang Road } \\
\text { Liu, Wei; Key Laboratory of Food Safety Research, Institute for Nutritional } \\
\text { Sciences, Shanghai Institutes for Biological Sciences, Chinese Academy of } \\
\text { Sciences, } 320 \text { Yue-yang Road } \\
\text { Zhao, Wei; } 2011 \text { Collaborative Innovation Center of Tianjin for Medical } \\
\text { Epigenetics, Key Laboratory of Hormones and Development (Ministry of } \\
\text { Health), Metabolic Diseases Hospital \& Tianjin Institute of Endocrinology, } \\
\text { Tianjin Medical University, } 66 \text { Tong-an Road } \\
\text { Zhang, Ya-jing; } 2011 \text { Collaborative Innovation Center of Tianjin for Medical } \\
\text { Epigenetics, Key Laboratory of Hormones and Development (Ministry of } \\
\text { Health), Metabolic Diseases Hospital \& Tianjin Institute of Endocrinology, } \\
\text { Tianjin Medical University, } 66 \text { Tong-an Road } \\
\text { Zhang, Hong; } 2011 \text { Collaborative Innovation Center of Tianjin for Medical } \\
\text { Epigenetics, Key Laboratory of Hormones and Development (Ministry of } \\
\text { Health), Metabolic Diseases Hospital \& Tianjin Institute of Endocrinology, } \\
\text { Tianjin Medical University, } 66 \text { Tong-an Road } \\
\text { Ying, Hao; Key Laboratory of Food Safety Research, Institute for Nutritional } \\
\text { Sciences, Shanghai Institutes for Biological Sciences, Chinese Academy of } \\
\text { Sciences, 320 Yue-yang Road; Key Laboratory of Food Safety Risk } \\
\text { Assessment, Ministry of Health }\end{array}$ \\
\hline Keyword: & $\begin{array}{l}\text { AMP-activated protein kinase, insulin signaling pathway, microRNA-29, } \\
\text { phosphoinositide 3-kinase, p85a }\end{array}$ \\
\hline
\end{tabular}

\section{SCHOLARONE ${ }^{m}$ \\ Manuscripts}




\title{
AICAR enhances insulin signaling via downregulation of
}

\section{miR-29}

\author{
Jia LIU ${ }^{1}$, Cheng YE ${ }^{2}$, Wei LIU ${ }^{2}$, Wei ZHAO ${ }^{1}$, Ya-jing ZHANG ${ }^{1}$, Hong ZHANG ${ }^{1, *}$ \\ and Hao YING ${ }^{2,3 *}$
}

1. 2011 Collaborative Innovation Center of Tianjin for Medical Epigenetics, Key Laboratory of Hormones and Development (Ministry of Health), Metabolic Diseases

Hospital \& Tianjin Institute of Endocrinology, Tianjin Medical University, 66 Tong-an Road, Tianjin 300070, China

2. Key Laboratory of Food Safety Research, Institute for Nutritional Sciences, Shanghai Institutes for Biological Sciences, Chinese Academy of Sciences, 320 Yue-yang Road, Shanghai 200031, China

3. Key Laboratory of Food Safety Risk Assessment, Ministry of Health, Beijing 100021, China.

\section{Corresponding author:}

Hong ZHANG: 2011 Collaborative Innovation Center of Tianjin for Medical Epigenetics, Key Laboratory of Hormones and Development (Ministry of Health), Metabolic Diseases Hospital \& Tianjin Institute of Endocrinology, Tianjin Medical University, 66 Tong-an Road, Tianjin 300070, China.

Hao YING: Key Laboratory of Food Safety Research, Institute for Nutritional Sciences, Shanghai Institutes for Biological Sciences, Chinese Academy of Sciences, 320

Yue-yang Road, Shanghai 200031, China, Email: yinghao@sibs.ac.cn. 


\section{Abstract}

As an activator of AMPK, AICAR's effect on insulin signaling has not been investigated extensively. PI3K/Akt is critical nodes involved in insulin signaling pathway. We observed that concomitant with the activation of AMPK by AICAR, the protein level of PI3K p85 $\alpha$ and the insulin-induced phosphorylation of Akt were enhanced in mouse primary hepatocytes. Previously, we identified a group of AMPK-regulated miRNAs in hepatocytes. It is not clear that whether miRNAs are related to the regulation of insulin signaling by AMPK. Here, we confirmed the negative regulation of miR-29 family members by AICAR treatment in mouse primary hepatocytes. Our results indicated that p85 $\alpha$ is a direct target of miR-29 and is negatively regulated by miR-29b in hepatocytes. In agreement with the findings in vitro, we found that the expression of miR-29 and the protein levels of p85 were inversely correlated in the liver of fasted mice.

Overexpression of miR-29b reduced the insulin-induced phosphorylation of Akt in hepatocytes, suggesting that miR-29 could serve as a negative regulator of insulin signaling. Furthermore, we found that overexpression of miR-29 could attenuate the effect of AICAR on $\mathrm{p} 85 \alpha$ expression. Taken together, our results indicated that activation of AMPK might enhance insulin signaling via downregulation of miR-29.

Keywords: AMP-activated protein kinase, insulin signaling pathway, microRNA-29, phosphoinositide 3-kinase, p85a 


\section{Introduction}

The activity of AMP-activated protein kinase (AMPK) is suppressed in disorders closely related to insulin resistance (Luo et al. 2005). 5-Aminoimidazole-4-carboxamide 1- $\beta$-D-ribofuranoside (AICAR), an AMPK activator, has been suggested in the regulation of hepatic glucose and lipid metabolism (Ruderman and Saha 2006; Zhou et al. 2001). Meanwhile, it has also been reported that the association of $p 85 \alpha$ subunit of phosphoinositide 3-kinase (PI3K) with IRS-1 and subsequent Akt phosphorylation were reduced in the liver of high fat-fructose diet-feeding mice due to AMPK activation (Yogalakshmi et al. 2014). However, whether and how AMPK signaling affects hepatic insulin signaling is largely unknown.

There are several reports discussing the roles of miRNAs in multiple metabolic processes, in which make it well acknowledged that miRNAs play great roles in the development and progression of metabolic diseases (Esau et al. 2006; Wang et al. 2013). While quite a few investigations (Godlewski et al. 2010a; Godlewski et al. 2010b) have reduced groups of miRNAs which show altered expression profiles under AMPK signaling pathway. Our laboratory carried out miRNA microarray study resently (Liu et al. 2013) has shown decreased levels of miR-29 family members in mouse primary hepatocytes treated with AICAR. Besides He et al. informed its role in diabetes (He et al. 2007), miR-29 is related to several physiopathology process including fibrosis, sclerosis, apoptosis, myogenesis, and tumorogenesis (Kapinas et al. 2009; Maurer et al. 2010; Mott et al. 2007; Wang et al. 2008). However, whether miR-29 is involved in the crosstalk between AMPK signaling and insulin signaling pathway is not clear. 
One of the critical nodes of insulin signaling pathway is PI3K, which is activated by binding of the $\mathrm{p} 85 \alpha$ regulatory subunit to specific sites on IRS1/IRS2 (Harrington et al. 2005). Recently, the role of miRNAs in the regulation of insulin signaling has been extensively studied (Karolina et al. 2011; Wang et al. 2014). For example, miR-378 inhibits hepatic insulin signaling through targeting p110 $\alpha$, a catalytic subunit of PI3K (Liu et al. 2014). It has been shown that miR-29 is able to enhance the gluconeogenesis through targeting hepatic $\mathrm{p} 85 \alpha$, and the expression of phosphoenolpyruvate carboxykinase (PEPCK) increased (Pandey et al. 2011). However, whether miR-29 could affect insulin-induced signaling transduction has not been investigated.

In this study, we observed that activation of AMPK by AICAR could increase insulin-induced phosphorylation of Akt as well as the protein levels of p85 $\alpha$ in mouse primary hepatocytes, suggesting that activation of AMPK signaling is able to amplify insulin signaling. Since we found that miR-29 levels were decreased in the AICAR-treated hepatocytes, we hypothesized that miR-29 might be involved in the regulation of insulin signaling by AMPK. Indeed, we found that miR-29 directly targets p85 and inhibits insulin-induced phosphorylation of Akt in hepatocytes. Thus this current study is a remarkable addition to the present knowledge of miRNAs in AMPK related insulin signaling pathway.

\section{Materials and Methods}

\section{Animal experiments}


Wild-type C57BL/6 mice (eight, male), purchased from Shanghai Laboratory Animal Center of the Chinese Academy of Sciences (Shanghai, China), were randomly divided into two groups with 4 mice per group, which were kept under standard conditions with 12 hours light/dark cycles and access to food and water ad libitum before 8 weeks old. One group of mice was fasted for $14 \mathrm{~h}$, and livers from both groups were immediately removed and snap-frozen in liquid nitrogen. All animal care and procedures were performed according to the instructions of 'The Animal Care and Welfare Committee' of the Peking Union Medical College (Beijing, China).

\section{Cell culture and transfection}

Primary mouse hepatocytes were isolated and cultured as previously described (Liu et al. 2013). Mouse Hepa1-6 hepatocytes were cultured as described (Djian et al. 1985). The miR-29 gain-of-function study was performed using miR-29b mimics (100 nM) and its negative control $(100 \mathrm{nM})$ on the C57BL/6 mouse primary hepatocytes. The miR-29b mimics sequence is 5'- UAGCACCAUUUGAAAUCAGUGUU -3'. Hepatocytes were transfected using lipofectamine ${ }^{\mathrm{TM}} 2000$ reagent (Invitrogen, USA) in DMEM (Gibco, USA), according to the manufacturer's instructions. The relative level of miR-29b in transfected hepatocytes was detected by qRT-PCR.

\section{Real-time quantitative PCR analysis}

\section{Real-time quantitative PCR analysis}

Total RNA extracted from cells was using Trizol reagent (Invitrogen, CA) and miRNeasy 
mini kit (QIAGEN) according to manufacturer's instructions. RNA quality and quantity was measured using nanodrop spectrophotometer (ND-1000, Nanodrop Technologies). RNA integrity was determined by gel electrophoresis. The samples were reverse-transcribed to cDNA using the reverse transcription kit (TaKaRa, Japan). The expression of mRNA was quantified with real-time PCR was carried out using SYBR Green qPCR mix. The sense and antisense PCR primers used, respectively, were as follows: for p85 $\alpha, 5$ '-GATTTTAATCTCAACGGAATGG-3', 5'-CTAGGTGTAAGAGTGTAATCGC-3'; peroxisome proliferator-activated receptor- $\gamma$ coactivator-1 $\alpha(\operatorname{Pgc}-1 \alpha)$, 5'-ATACCGCAAAGAGCACGAGAAG-3', 5'-CTCAAGAGCAGCGAAAGCGTCACAG-3'; for Pepck, 5'-GTGCTGGAGTGGATGTTCGG-3', 5'-CTGGCTGATTCTCTGTTTCAGG-3'; for glucose-6-phosphase (G6pase), 5'-ACTGTGGGCATCAATCTCCTC-3', 5'-CGGGACAGACAGACGTTCAGC-3'; and for $\beta$-actin, 5'-TGGAATCCTGTGGCATCCATGAAAC -3', 5'-TAAAACGCAGCTCAGTAACAGTCCG -3'. miR-29 family's relative expression was calculated using the expression of U6 small nuclear RNA as the reference. The sequence-specific forward primers for mature miR-29 and U6 internal control were as follows: for miR-29a, 5'- UAGCACCAUCUGAAAUCGGUUA -3'; for miR-29b, 5'-UAGCACCAUUUGAAAUCAGUGUU -3'; for miR-29c, 5'-UAGCACCAUUUGAAAUCGGUUA -3'; and for U6, 5'-CTCGCTTCGGCAGCACA-3'.

\section{Western blotting analysis}


Primary mouse hepatocytes were treated with AICAR or miR-29b mimics. Cultured hepatocytes were then harvested and extracted in RIPA lysis buffer. The protein samples were separated by SDS-PAGE gels and transferred to nitrocellulose membrane. The membranes were washed with Tris-buffered saline supplemented with $0.05 \%$ NP-40 and $5 \%$ nonfat dry milk for $30 \mathrm{~min}$ at $37^{\circ} \mathrm{C}$. Immunoblotting was performed following standard procedures, and the signals were detected using chemiluminescence reagents (Syngene). Primary antibodies were directed against: p-AMPK, p-Akt, p85 $\alpha$, $\beta$-actin and tubulin (all from Santa Cruz).

\section{Statistical analysis}

Results were presented as mean \pm standard deviation. Statistical analyses were assessed using $T$-test between two groups and one-way ANOVA with multiple groups. All analyses were performed with SPSS 19.0 (SPSS Inc., USA), and a value of $P<0.05$ was considered to indicate statistical significance.

\section{Results}

\section{Effects of AICAR on insulin signaling}

AICAR is a specific activator of AMPK. The phosphorylation of AMPK increased significantly after AICAR treatment (Fig. 1A). We first investigated the effect of AICAR on insulin signaling transduction in $\mathrm{C} 57 \mathrm{BL} / 6$ mouse primary hepatocytes. As shown in Fig.1B, the insulin-induced phosphorylation of S473 on Akt in absence or presence of 
AICAR $(0.5 \mathrm{mM}, 2 \mathrm{~h})$ was examined in primary hepatocytes. Interestingly, we observed that AICAR augmented the insulin-stimulated phosphorylation of Akt at S473 by more than 2-fold (Fig.1B), indicating that activation of AMPK signaling might enhance insulin signaling in liver cells.

$\mathrm{P} 85 \alpha$ is the regulatory subunit of $\mathrm{PI} 3 \mathrm{~K}$, which is important in insulin signaling pathway and an upstream protein of Akt. To evaluate whether AICAR treatment has any effects on p85 $\alpha$ expression, C57BL/6 mouse primary hepatocytes were treated with AICAR followed by insulin administration, and the p $85 \alpha$ protein level were examined. As shown in Fig.1C, AICAR treatment caused an increase of $\mathrm{p} 85 \alpha$ protein levels both in the presence and absence of insulin. These results suggested that activation of AMPK signaling might be able to enhance insulin signaling pathway by elevating the protein expression levels of $\mathrm{p} 85 \alpha$.

\section{Effects of AICAR on miR-29 family in mouse primary hepatocytes}

Recently our laboratory carried out a miRNA microarray study in control versus C57BL/6 mouse primary hepatocytes (Liu et al. 2013) showed decreased levels of all miR-29 family members in AICAR-treated hepatocytes. To verify this result, real time PCR was performed to detect the expression of miR-29a, miR-29b and miR-29c in C57BL/6 mouse primary hepatocytes after AICAR treatment. AICAR concentrations 0.2 $\mathrm{mM}$ and $0.4 \mathrm{mM}$ were chosen to the concentrations described (Cacicedo et al. 2004) to examine whether the treatment of AICAR influenced the expression of miR-29a, miR-29b and miR-29c. As shown in Fig.2, dibutyryl-cAMP (Bt ${ }_{2}$-cAMP) treatment 
increased the expression of all three miR-29 family members significantly in hepatocytes. Following AICAR treatment, the expression of miR-29a, miR-29b and miR-29c were all decreased in a dose-dependent manner, suggesting that all miR-29 family members might be involved in AMPK signaling pathway (Fig.2).

\section{miR-29 targets p85 $\alpha$ regulated insulin signaling in C57BL/6 mouse}

\section{primary hepatocytes}

PI3K p85 $\alpha$ subunit had been reported as a target of miR-29 in a hepatic cell line (Park et al. 2009). However, whether miR-29 could affect the insulin-induced phosphorylation of Akt was not clear, which requires further study. In this study, we first determined whether miR-29 overexpression could decrease the protein level of p85a in primary hepatocytes. Since the three homologs of miR-29 family have the same 'seed sequence' and the effects of AICAR on miR-29b were more evident, miR-29b was chosen to contrive miRNA mimics. As shown in Fig. 3B, the real time PCR results indicated that the transfection of miR-29b mimics effectively elevated the expression of miR-29b in primary hepatocytes $(P<0.01)$. In agreement with the previous report, overexpression of miR-29b resulted in a decrease in both mRNA and protein levels of $\mathrm{p} 85 \alpha(P<0.05)$ (Fig. 3C and D).

To evaluated the downstream of PI3K in insulin signaling pathway, we then detected the gluconeogenesis related gene Pgc-1 $\alpha$, Pepck and G6Pase expression after miR-29b mimics treatment. The real time PCR results indicated that the expression of Pgc-1 $\alpha$, Pepck and G6Pase were elevated significantly after the transfection of miR-29b mimics in primary hepatocytes $(P<0.01)$ (Fig. 3E). 


\section{The expression of miR-29 and p85 $\alpha$ in the liver of fasted C57BL/6 mice}

Since our results suggested that $\mathrm{p} 85 \alpha$ is a direct target of miR-29, we then examined whether the expression levels of miR-29 family members and p85a protein levels are conversely correlated in the liver of mice under different feeding conditions. First of all, we compared the abundance of each miR-29 family members in the liver of C57BL/6 mice and primary hepatocytes. As shown in Fig. 4A, the expression of miR-29a was slightly higher than that of miR-29b or miR-29c (Fig. 4A). Interestingly, we found that the expression levels of all three miR-29 family members increased significantly in the liver of mice during fasting (Fig. 4B). In contrast, both of mRNA and protein levels of p85a decreased significantly in the liver of mice upon fasting. These in vivo results further supported the notion that $\mathrm{p} 85 \alpha$ is a target of miR-29. Insulin receptor- $\beta$ increased, and its downstream the phosphorylation of insulin-receptor, substrate IRS1, Akt reduced in skeletal muscle biopsies of volunteer subjects after fasting (Wijngaarden et al. 2013). Our data also indicated that the elevation of miR-29 expression and the repressed p85 $\alpha$ protein expression might facilitate the inhibition of insulin signaling in fasted status.

\section{Effects of miR-29b on insulin signaling}

We then assessed the effect of miR-29b mimics on insulin-induced phosphorylation of S473 on Akt in C57BL/6 mouse primary hepatocytes. As shown in Fig. 5A, the miR-29b mimics incubation markedly inhibited insulin-induced Akt phosphorylation $(P<0.01)$. Similar results were obtained in Hepa1-6 hepatocytes. These results suggested that miR-29b was able to inhibit insulin signaling. 


\section{MiR-29 diminishes the effect of AICAR on the expression of p85a}

Based on our findings, we hypothesized that the repression of miR-29 by AICAR, which leads to the derepression of $\mathrm{p} 85 \alpha$, might be involved in the augment of insulin signaling by AICAR. To further test this hypothesis, we investigated whether overexpression of miR-29b could antagonize the effect of AICAR on p85a. As shown in Fig. 6, AICAR treatment enhanced the protein expression level of p85a, which could be repressed by overexpression of miR-29b. These results further supported our hypothesis that miR-29b might mediate the effects of AICAR on insulin signaling.

\section{Discussion}

In previously study, we found that the expression levels of all of the miR-29 family members were significantly decreased in AICAR treated C57BL/6 mouse primary hepatocytes compared with normal control (Liu et al. 2013), implying that miR-29 family members might be involved in hepatic AMPK signaling pathway. In mice, either the miR-29b-1/miR-29a cluster or the miR-29b-2/miR29c cluster encodes the mice's three miR-29 paralogs, miR-29a, miR-29b and miR-29c (Hwang et al., 2007). In this research, the expression of miR-29a, miR-29b and miR-29c were all decreased in a dose-dependent manner, suggesting that all the miR-29 family members might be involved in AMPK signaling pathway.

Complex relationship exists between the AMPK and insulin signaling pathways. It was reported that AMPK regulate the activation IRS-1 (Tzatsos and Tsichlis 2007) and Akt 
(Levine et al. 2007), while AMPK activation is negatively impacted by insulin and Akt in liver (Hahn-Windgassen et al. 2005; Qiao et al. 2002). We observed that AICAR augmented the insulin-stimulated phosphorylation of Akt at S473 by more than 2-fold (Fig.1A), indicating that activation of AMPK signaling might enhance insulin signaling in liver cells, which was the same with previous research (Tao et al. 2010). p85a is the regulatory subunit of $\mathrm{PI} 3 \mathrm{~K}$, which is important in insulin signaling pathway and a direct upstream protein of Akt. AICAR treatment caused an increase of p85 $\alpha$ protein levels both in the presence and absence of insulin, which suggested that activation of AMPK signaling might be able to enhance insulin signaling pathway by elevating the protein expression level of $\mathrm{p} 85 \alpha$.

He et al. found that the increase in miR-29 level caused insulin resistance, resemble that of incubation with high glucose and insulin. And high glucose and insulin may induced the expression of miR-29a and miR-29b (He et al. 2007). These results suggested that miR-29 family were related to glucose metabolism. We searched miR-29 family's targets with TargetScan v5.2 (http://www.targetscan.org ) that the p85a regulatory subunit of PI3K binding sites for miR-29 at positions of its 3'UTR 331 337 (Fig. 3A). And it is conserved across species. It was validated in Hela cells by Park et al. that led to negative regulation of the tumor suppressor protein, p53 (Park et al. 2009), and in HepG2 cells by Panday et al. that prevented insulin stimulated Akt phosphorylation even in the presence of unaltered total Akt levels. We speculated that AMPK might increase insulin signaling pathway by miR-29 targeting p $85 \alpha$. In this study, we first determined whether miR-29 overexpression could decrease the protein level of $\mathrm{p} 85 \alpha$ in primary hepatocytes. Since the 
three homolog of miR-29 family have the same 'seed sequence' and the effects of AICAR on miR-29b was more evident, miR-29b was chosen to contrive miRNA mimics.

MiR-29b mimics mediated overexpression of miR-29b in mouse primary hepatocytes could largely decrease both mRNA and protein levels of $\mathrm{p} 85 \alpha$. Liang et al. found that overexpression of miR-29a-c in hepatocytes decreased the protein levels of Pgc-1 $\alpha$ and G6Pase, and hence reduced hepatocytes and hepatic glucose production (Liang et al. 2013). And our results showed that the gluconeogenesis related gene Pgc-1 $\alpha$, Pepck and G6Pase were increased after miR-29b mimics treatment, which was consistent with those results. All of these results indicated that $\mathrm{p} 85 \alpha$ is a direct target of miR-29 and is negatively regulated by miR-29b in hepatocytes (Fig. 3).miR-29 inhibited PI3K pathway, and elevated the activation of gluconeogenesis.

We then compared the abundance of each miR-29 family members in the liver of C57BL/6 mice and primary hepatocytes. As shown in Fig. 4A, the expression of miR-29a was slightly higher than that of miR-29b or miR-29c. We found that the expression levels of all the three miR-29 family members significantly increased in the liver of mice during fasting (Fig. 4B). In contrast, both of mRNA and protein levels of p85a decreased significantly in the liver of mice upon fasting. Since the expression levels of miR-29 family members and $\mathrm{p} 85 \alpha$ protein level were always conversely correlated in the liver of mice under different feeding conditions, these in vivo results further supported the notion that $\mathrm{p} 85 \alpha$ is a target of miR-29. Insulin signaling is repressed during fasting. Insulin receptor- $\beta$ increased, and its downstream the phosphorylation of insulin-receptor, substrate IRS1, Akt reduced in skeletal muscle biopsies of volunteer subjects after fasting 
(Wijngaarden et al. 2013). These results also indicated that the elevation of miR-29 expression and the repressed $\mathrm{p} 85 \alpha$ protein expression might facilitate the inhibition of insulin signaling in fasted status.

More importantly, miR-29 could repress the insulin-induced phosphorylation of Akt both in primary hepatocytes and mice Hepa1-6 hepatocytes, which suggested that miR-29b mimics was able to inhibit insulin signaling pathway. Further research showed that AICAR treatment enhanced the protein expression level of $\mathrm{p} 85 \alpha$, which could be repressed by overexpression of miR-29b. These results further supported our hypothesis that miR-29 leads to the derepression of $\mathrm{p} 85 \alpha$, which might mediate the effects of AICAR on insulin signaling (Fig. 6). This seems opposite to that of Liang et al. We speculated that it was because treatment of dexamethasone may mimic the pathologic station of insulin resistance. miR-29 may have different effects in physical and pathologic stations, which needs further investigation.

In this research, we conformed that AICAR treatment decreased the expression of all the miR-29 family members in C57BL/6 mouse primary hepatocytes, activated the PI3K-Akt pathway, hence insulin signaling pathway. AICAR treatment enhanced the protein level of p85a, which could be repressed by overexpression miR-29b. These results further supported our hypothesis that miR-29 leads to the derepression of $\mathrm{p} 85 \alpha$, which might mediate the effects of AICAR on insulin signaling. The in vivo results indicated that the elevation of miR-29 expression and the repressed p85a protein level might facilitate the inhibition of insulin signaling in fasted status, which would be good for maintaining the 
glucose homeostasis. Taken together, our results indicated that activation of AMPK might enhance insulin signaling via downregulation of miR-29.

Abbreviations AICAR, 5-aminoimidazole-4-carboxamide-1- $\beta$-d-ribofuranoside; Akt/PKB, serine-threonine protein kinase; AMPK, AMP-activated protein kinase; $\mathrm{Bt}_{2}$-cAMP, dibutyryl-cAMP; IRS-1, insulin receptor substance 1; miRNAs, microRNAs; PI3K, phosphoionositide-3-kinase.

\section{Acknowledgements}

This work was supported by grants from the National Natural Science Foundation (No. 81400784, 31371189) and Shanghai Institutes for Biological Sciences, Chinese Academy of Sciences (SIBS2012004).

\section{Disclosure}

None declared.

\section{References}

Cacicedo, J.M., Yagihashi, N., Keaney, J.F., Jr., Ruderman, N.B., and Ido, Y. 2004. AMPK inhibits fatty acid-induced increases in NF-kappaB transactivation in cultured human umbilical vein endothelial cells. Biochem. Biophys. Res. Commun. 324(4): 1204-1209. PMID: 15504342.

Djian, P., Phillips, M., and Green, H. 1985. The activation of specific gene transcription in the adipose conversion of 3T3 cells. J. Cell Physiol. 124(3): 554-556. PMID: 4044664. 
Esau, C., Davis, S., Murray, S.F., Yu, X.X., Pandey, S.K., Pear, M., et al. 2006. miR-122 regulation of lipid metabolism revealed by in vivo antisense targeting. Cell Metab. 3(2): 87-98. PMID: 16459310.

Godlewski, J., Bronisz, A., Nowicki, M.O., Chiocca, E.A., and Lawler, S. $2010 a$. microRNA-451: A conditional switch controlling glioma cell proliferation and migration. Cell Cycle. 9(14): 2742-2748. PMID: 20647762.

Godlewski, J., Nowicki, M.O., Bronisz, A., Nuovo, G., Palatini, J., De Lay, M., et al. 2010b. MicroRNA-451 regulates LKB1/AMPK signaling and allows adaptation to metabolic stress in glioma cells. Mol. Cell, 37(5): 620-632. PMID: 20227367.

Hahn-Windgassen, A., Nogueira, V., Chen, C.C., Skeen, J.E., Sonenberg, N., and Hay, N. 2005. Akt activates the mammalian target of rapamycin by regulating cellular ATP level and AMPK activity. J. Biol. Chem. 280(37): 32081-32089. PMID: 16027121. Harrington, L.S., Findlay, G.M., and Lamb, R.F. 2005. Restraining PI3K: mTOR signalling goes back to the membrane. Trends Biochem. Sci. 30(1): 35-42. PMID: 15653324.

He, A., Zhu, L., Gupta, N., Chang, Y., and Fang, F. 2007. Overexpression of micro ribonucleic acid 29, highly up-regulated in diabetic rats, leads to insulin resistance in 3T3-L1 adipocytes. Mol. Endocrinol. 21(11): 2785-2794. PMID: 17652184.

Kapinas, K., Kessler, C.B., and Delany, A.M. 2009. miR-29 suppression of osteonectin in osteoblasts: regulation during differentiation and by canonical Wnt signaling. J. Cell Biochem. 108(1): 216-224. PMID: 19565563.

Karolina, D.S., Armugam, A., Tavintharan, S., Wong, M.T., Lim, S.C., Sum, C.F., et al. 2011. MicroRNA 144 impairs insulin signaling by inhibiting the expression of insulin 
receptor substrate 1 in type 2 diabetes mellitus. PLoS One, 6(8): e22839. PMID: 21829658.

Levine, Y.C., Li, G.K., and Michel, T. 2007. Agonist-modulated regulation of AMP-activated protein kinase (AMPK) in endothelial cells. Evidence for an AMPK -> Rac1 -> Akt -> endothelial nitric-oxide synthase pathway. J. Biol. Chem. 282(28): 20351-20364. PMID: 17519230.

Liang, J., Liu, C., Qiao, A., Cui, Y., Zhang, H., Cui, A., et al. 2013. MicroRNA-29a-c decrease fasting blood glucose levels by negatively regulating hepatic gluconeogenesis. J. Hepatol. 58(3): 535-542. PMID: 23111009.

Liu, J., Liu, W., Ying, H., Zhao, W., and Zhang, H. 2013. Analysis of microRNA expression profile induced by AICAR in mouse hepatocytes. Gene, 512(2): 364-372. PMID: 23107762.

Liu, W., Cao, H., Ye, C., Chang, C., Lu, M., Jing, Y., et al. 2014. Hepatic miR-378 targets p1 $10 \alpha$ and controls glucose and lipid homeostasis by modulating hepatic insulin signalling. Nat. Commun. 5: 5682. PMID: 25471065.

Luo, Z., Saha, A.K., Xiang, X., and Ruderman, N.B. 2005. AMPK, the metabolic syndrome and cancer. Trends. Pharmacol. Sci. 26(2): 69-76. PMID: 15681023. Maurer, B., Stanczyk, J., Jungel, A., Akhmetshina, A., Trenkmann, M., Brock, M., et al. 2010. MicroRNA-29, a key regulator of collagen expression in systemic sclerosis. Arthritis Rheum. 62(6): 1733-1743. PMID: 20201077. Mott, J.L., Kobayashi, S., Bronk, S.F., and Gores, G.J. 2007. mir-29 regulates Mcl-1 protein expression and apoptosis. Oncogene, 26(42): 6133-6140. PMID: 17404574. Pandey, A.K., Verma, G., Vig, S., Srivastava, S., Srivastava, A.K., and Datta, M. 2011. 
miR-29a levels are elevated in the $\mathrm{db} / \mathrm{db}$ mice liver and its overexpression leads to attenuation of insulin action on PEPCK gene expression in HepG2 cells. Mol. Cell Endocrinol. 332(1-2): 125-133. PMID: 20943204.

Park, S.Y., Lee, J.H., Ha, M., Nam, J.W., and Kim, V.N. 2009. miR-29 miRNAs activate p53 by targeting p85 alpha and CDC42. Nat. Struct. Mol. Biol. 16(1): 23-29. PMID: 19079265.

Qiao, L.Y., Zhande, R., Jetton, T.L., Zhou, G., and Sun, X.J. 2002. In vivo phosphorylation of insulin receptor substrate 1 at serine 789 by a novel serine kinase in insulin-resistant rodents. J. Biol. Chem. 277(29): 26530-26539. PMID: 12006586. Ruderman, N.B., and Saha, A.K. 2006. Metabolic syndrome: adenosine monophosphate-activated protein kinase and malonyl coenzyme A. Obesity (Silver Spring), 14 Suppl 1: 25S-33S. PMID: 16642960.

Tao, R., Gong, J., Luo, X., Zang, M., Guo, W., Wen, R., et al. 2010. AMPK exerts dual regulatory effects on the PI3K pathway. J. Mol. Signal, 5(1): 1. PMID: 20167101. Tzatsos, A., and Tsichlis, P.N. 2007. Energy depletion inhibits phosphatidylinositol 3-kinase/Akt signaling and induces apoptosis via AMP-activated protein kinase-dependent phosphorylation of IRS-1 at Ser-794. J. Biol. Chem. 282(25): 18069-18082. PMID: 17459875.

Wang, H., Garzon, R., Sun, H., Ladner, K.J., Singh, R., Dahlman, J., et al. 2008. NF-kappaB-YY1-miR-29 regulatory circuitry in skeletal myogenesis and rhabdomyosarcoma. Cancer Cell, 14(5): 369-381. PMID: 18977326.

Wang, Y., Hu, C., Cheng, J., Chen, B., Ke, Q., Lv, Z., et al. 2014. MicroRNA-145 suppresses hepatocellular carcinoma by targeting IRS1 and its downstream Akt signaling. 
Biochem. Biophys. Res. Commun. 446(4): 1255-1260. PMID: 24690171.

Wang, Y.T., Tsai, P.C., Liao, Y.C., Hsu, C.Y., and Juo, S.H. 2013. Circulating

microRNAs have a sex-specific association with metabolic syndrome. J. Biomed. Sci.

4(20): 72. PMID:

Wijngaarden, M.A., van der Zon, G.C., van Dijk, K.W., Pijl, H., and Guigas, B. 2013.

Effects of prolonged fasting on AMPK signaling, gene expression, and mitochondrial respiratory chain content in skeletal muscle from lean and obese individuals. Am. J.

Physiol. Endocrinol. Metab. 304(9): E1012-1021. PMID: 23512807.

Yogalakshmi, B., Bhuvaneswari, S., Sreeja, S., and Anuradha, C.V. 2014. Grape seed proanthocyanidins and metformin act by different mechanisms to promote insulin signaling in rats fed high calorie diet. J. Cell Commun. Signal, 8(1): 13-22. PMID: 24026800 .

Zhou, G., Myers, R., Li, Y., Chen, Y., Shen, X., Fenyk-Melody, J., et al. 2001. Role of AMP-activated protein kinase in mechanism of metformin action. J. Clin. Invest. 108(8): 1167-1174. PMID: 11602624. 


\section{Figure captions}

Figure 1 Effects of AICAR on insulin signaling. (A) Expression levels of phosphorylation of AMPK in hepatocytes incubated with or without AICAR. (B) Expression levels of p-Akt in hepatocytes incubated with or without insulin or AICAR as indicated. (C) Protein expression levels of $\mathrm{p} 85 \alpha$ in hepatocytes incubated with or without insulin or AICAR as indicated.

Figure 2 Effects of AICAR on the expression of miR-29 family members in mouse primary hepatocytes. Relative expression of miR-29 family members was determined in C57BL/6 mouse hepatocytes incubated with different doses of AICAR ( $0.2 \mathrm{mM}$ or 0.4 $\mathrm{mM}$ for $6 \mathrm{~h})$. Results are representative of 3 independent experiments. Data were mean \pm SD. $* P<0.01$ compared with hepatocytes incubated without $\mathrm{Bt}_{2}$-cAMP; $\# P<0.01$ compared with hepatocytes incubated with $\mathrm{Bt}_{2}$-cAMP alone. Dex is short for dexamethasone.

Figure 3 miR-29b targets p85 $\alpha$ in C57BL/6 mouse primary hepatocytes. (A) Binding site of mmu-miR-29b on the 3 'UTR of the $p 85 \alpha$ regulatory subunit of PI3K. The seed region of miR-29b is complementary to residues 331 337 on the 3'UTR. Sequence alignment of the 3'UTR's of the p85 $\alpha$ subunit of PI3K across species. The conserved miR-29b seed region is shown within the white box. (B) The transfection of miR-29b mimics increased the expression of miR-29b (n=3). (C) Relative mRNA levels of $\mathrm{p} 85 \alpha$ expressed as fold activation after miR-29b mimics transfection $(n=3)$. (D) Western blot analysis was performed by using $\mathrm{p} 85 \alpha$ antibody in primary hepatocytes transfected with 
miR-29b mimics. (E) Relative mRNA levels of Pgc-1 $\alpha$, Pepck and G6Pase expressed elevated after miR-29b mimics transfection $(n=3) .{ }^{*} P<0.05, * * P<0.01$ compared with control.

Figure 4 Expression of miR-29 family and p85 $\alpha$ under fasting and fed conditions in C57BL/6 mouse liver. (A) There were no significant differences between the expression of miR-29 family members in the liver of C57BL/6 mice and primary hepatocytes $(n=3)$. (B) The expression of miR-29 family members increased significantly in the liver of fasted C57BL/6 mice ( $n=3-5)$. (C) Relative mRNA levels of $\mathrm{p} 85 \alpha$ in the liver of fasted mice $(n=3)$. (D) Protein levels of $\mathrm{p} 85 \alpha$ in the liver of fasted mice $(n=3)$. Data was mean \pm SD. ${ }^{*} P<0.05, * * P<0.01$ compared with control.

Figure 5 Effects of miR-29 on insulin signaling. (A) Expression levels of p-Akt in C57BL/6 mouse primary hepatocytes incubated with or without insulin or miR-29b mimics. (B) Expression levels of p-Akt in Hepa 1-6 hepatocytes incubated with or without insulin or miR-29b mimics.

Figure 6 Effects of AICAR and miR-29 mimics on hepatocytes expression of p85 $\alpha$. Expression levels of $\mathrm{p} 85 \alpha$ in hepatocytes incubated with or without miR-29b mimics and AICAR. 


\title{
A AICAR
}

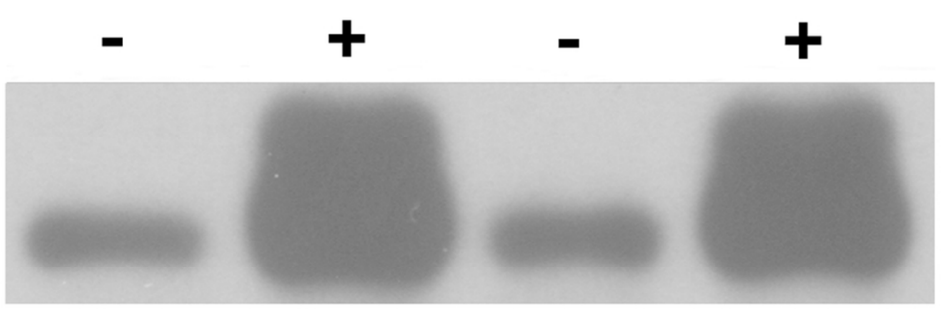

\section{$\beta$-actin}

Insulin
AICAR

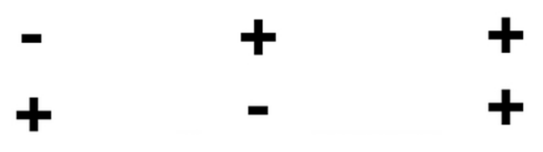

p-Akt

\author{
$\beta$-actin
}

\section{Insulin AICAR \\ p85 $\alpha$}

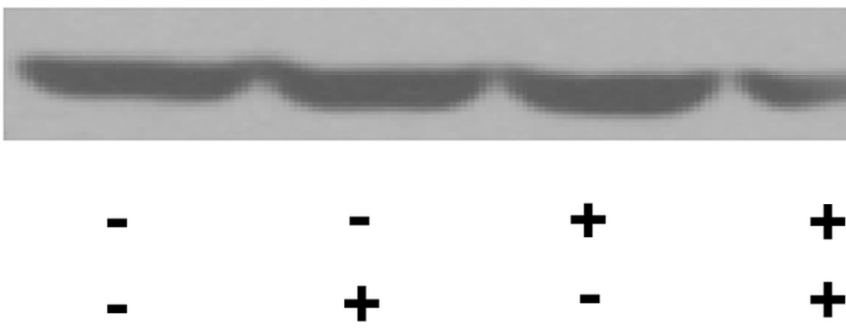

\section{$\beta$-actin}

Figure 1 Effects of AICAR on insulin signaling. (A) Expression levels of phosphorylation of AMPK in hepatocytes incubated with or without AICAR. (B) Expression levels of p-Akt in hepatocytes incubated with or without insulin or AICAR as indicated. (C) Protein expression levels of p85a in hepatocytes incubated with or without insulin or AICAR as indicated.

$99 \times 119 \mathrm{~mm}(300 \times 300 \mathrm{DPI})$ 

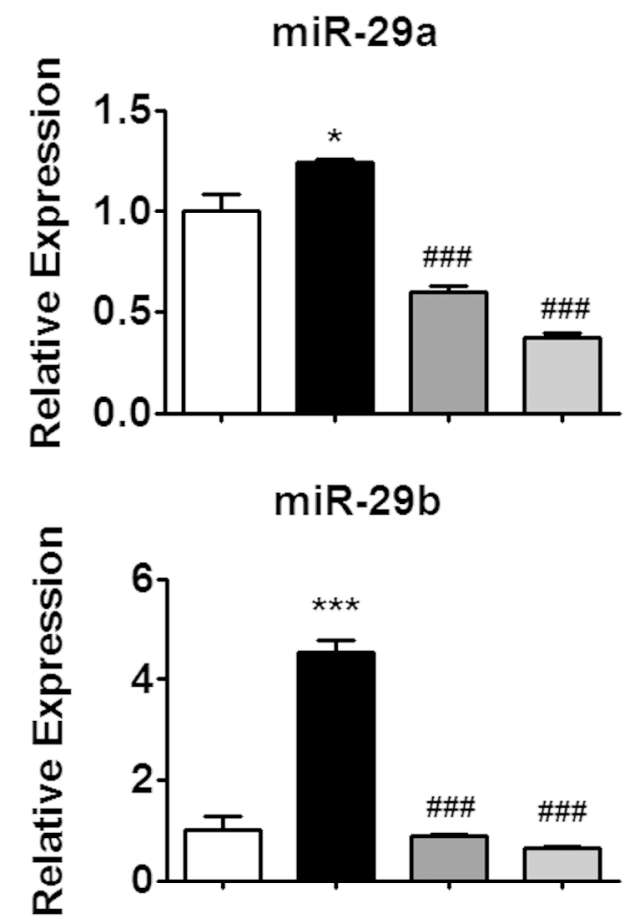

\section{miR-29c}

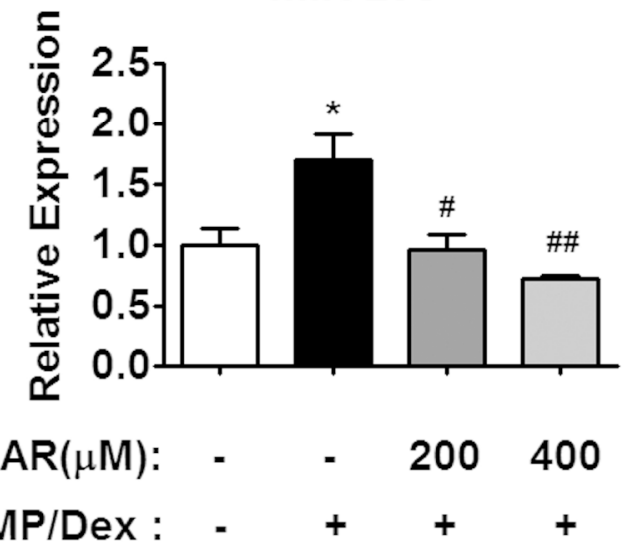

Figure 2 Effects of AICAR on the expression of miR-29 family members in mouse primary hepatocytes. Relative expression of miR-29 family members was determined in C57BL/6 mouse hepatocytes incubated with different doses of AICAR $(0.2 \mathrm{mM}$ or $0.4 \mathrm{mM}$ for $6 \mathrm{~h})$. Results are representative of 3 independent experiments. Data were mean $\pm \mathrm{SD}$. $* P<0.01$ compared with hepatocytes incubated without $\mathrm{Bt}_{2}-\mathrm{CAMP} ; \# P$ $<0.01$ compared with hepatocytes incubated with $\mathrm{Bt}_{2}$-cAMP alone. Dex is short for dexamethasone. $175 \times 377 \mathrm{~mm}(300 \times 300 \mathrm{DPI})$ 

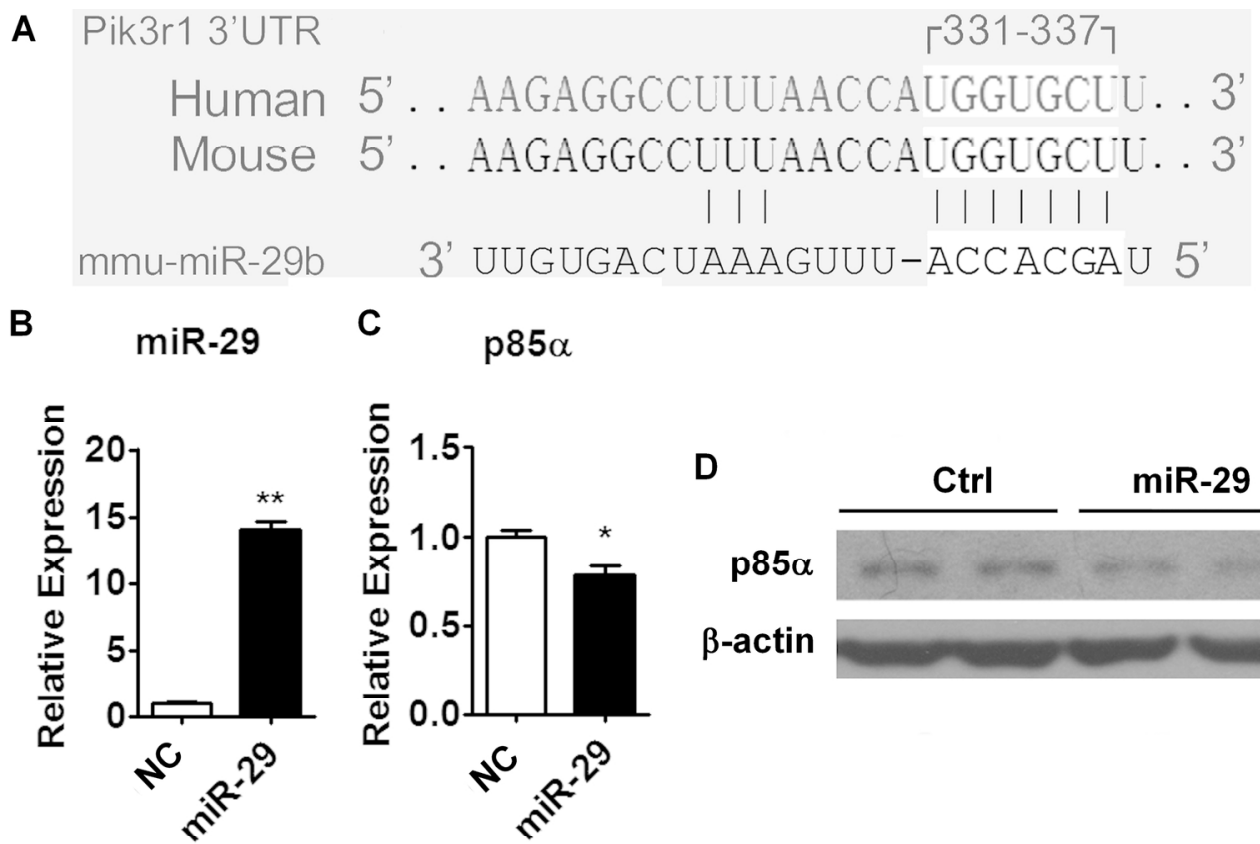

D

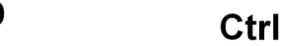
miR-29

$\mathrm{E}$

PGC-1a

PEPCK

G6Pase
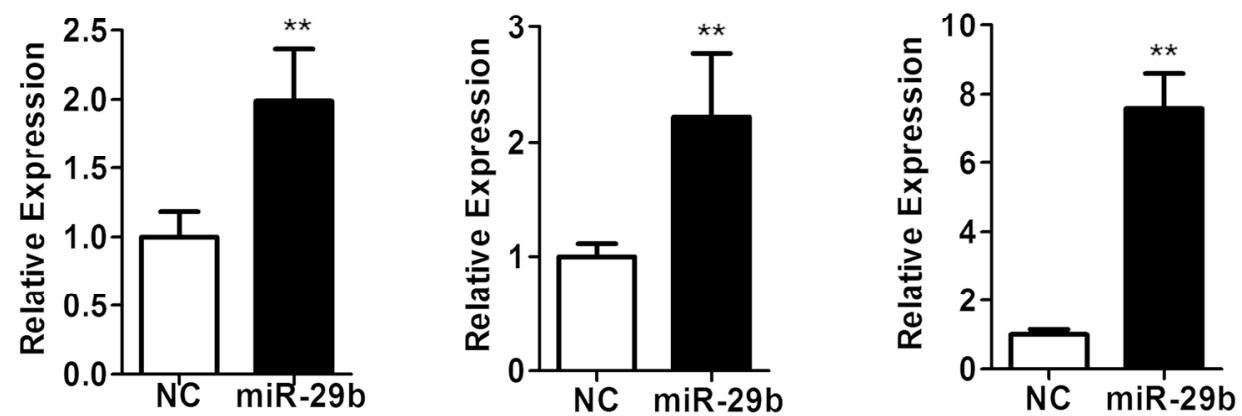

Figure 3 miR-29b targets p85a in C57BL/6 mouse primary hepatocytes. (A) Binding site of mmu-miR-29b on the 3 'UTR of the p85a regulatory subunit of PI3K. The seed region of miR-29b is complementary to residues 331 337 on the $3^{\prime} U T R$. Sequence alignment of the $3^{\prime} U$ TRR' $^{\prime}$ of the p85a subunit of PI3K across species. The conserved miR-29b seed region is shown within the white box. (B) The transfection of miR-29b mimics increased the expression of miR-29b $(n=3)$. (C) Relative mRNA levels of p85a expressed as fold activation after miR-29b mimics transfection ( $n=3$ ). (D) Western blot analysis was performed by using p85a antibody in primary hepatocytes transfected with miR-29b mimics. (E) Relative mRNA levels of Pgc-1a, Pepck and G6Pase expressed elevated after miR-29b mimics transfection $(n=3) . * P<0.05, * * P<0.01$ compared with control.

$175 \times 184 \mathrm{~mm}(300 \times 300 \mathrm{DPI})$ 

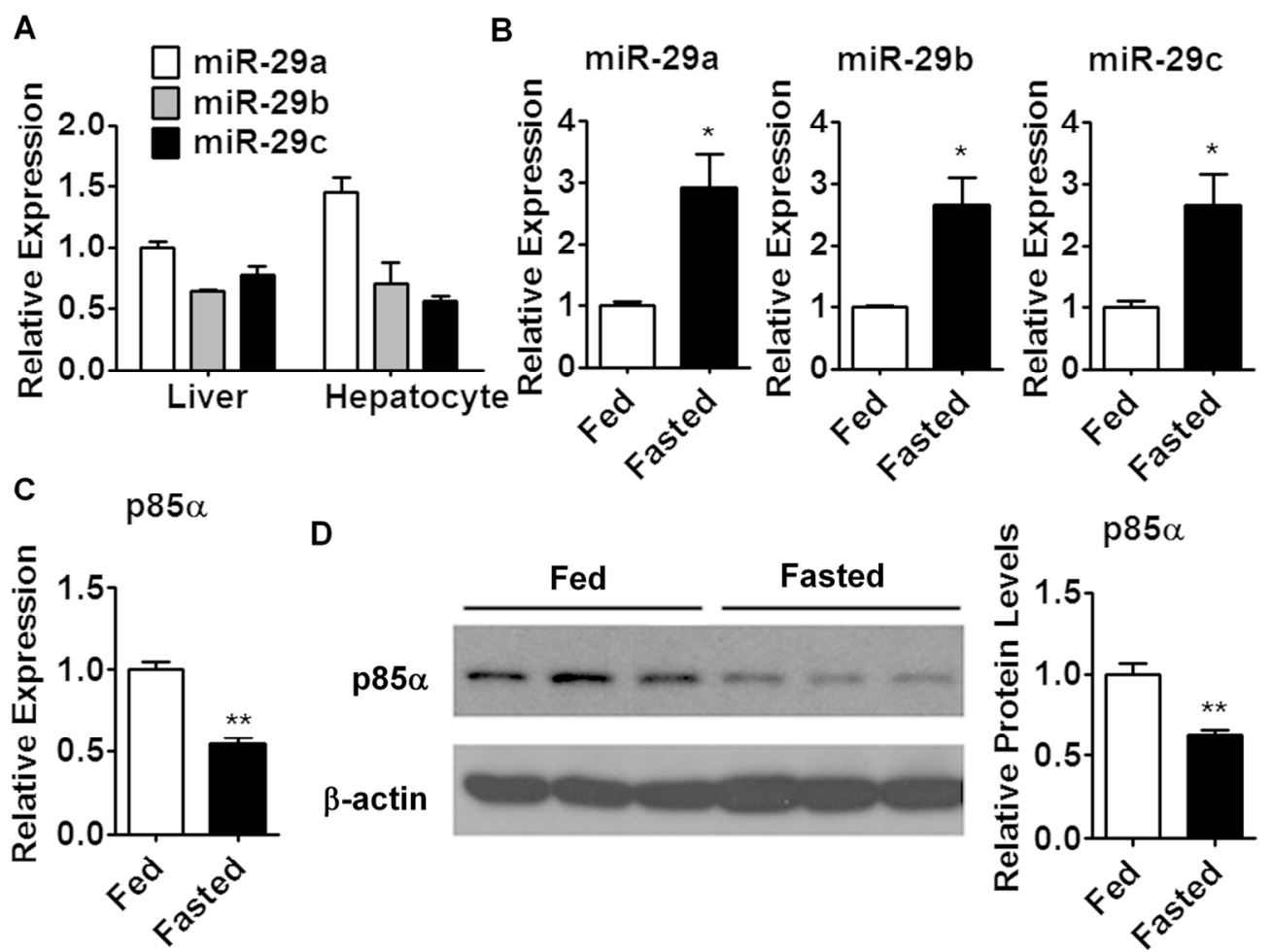

D

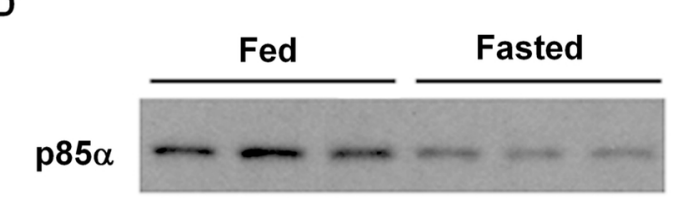

$\beta$-actin

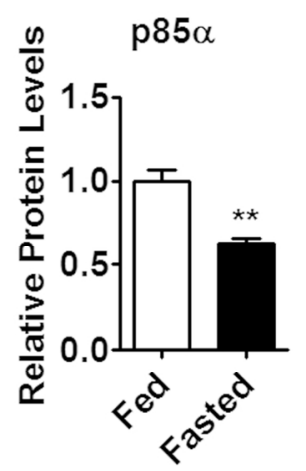

Figure 4 Expression of miR-29 family and p85a under fasting and fed conditions in C57BL/6 mouse liver. (A) There were no significant differences between the expression of miR-29 family members in the liver of C57BL/6 mice and primary hepatocytes $(n=3)$. (B) The expression of miR-29 family members increased significantly in the liver of fasted C57BL/6 mice $(n=3-5)$. (C) Relative mRNA levels of p85a in the liver of fasted mice $(n=3)$. (D) Protein levels of p85a in the liver of fasted mice $(n=3)$. Data was mean \pm SD. $* P<$ $0.05, * * P<0.01$ compared with control. $134 \times 103 \mathrm{~mm}(300 \times 300 \mathrm{DPI})$ 


\section{A Mimics NC miR-29 NC miR-29 Insulin - $\quad-\quad+\quad+$} p-Akt Tubulin

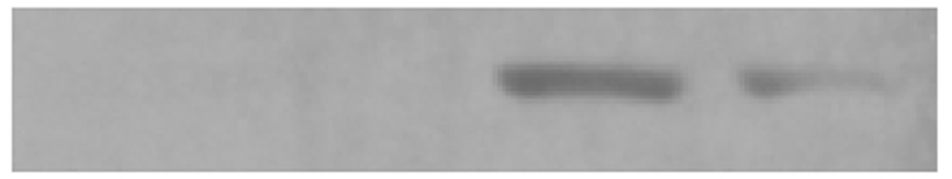

B

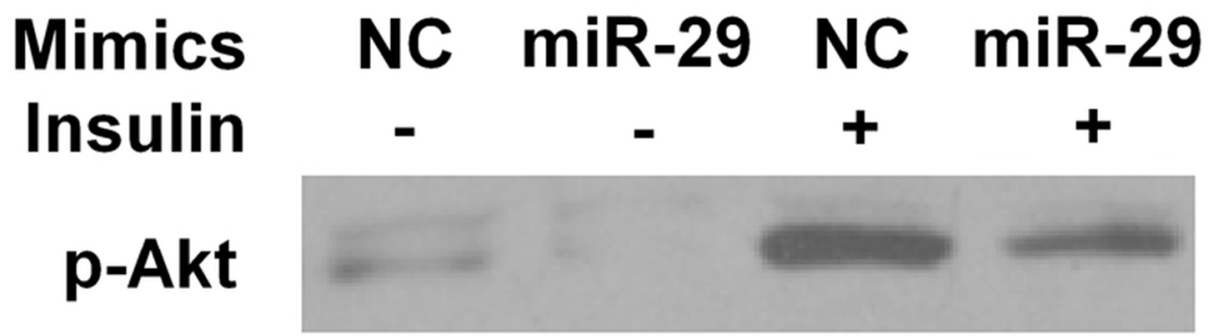
$\beta$-actin

Figure 5 Effects of miR-29 on insulin signaling. (A) Expression levels of p-Akt in C57BL/6 mouse primary hepatocytes incubated with or without insulin or miR-29b mimics. (B) Expression levels of p-Akt in Hepa 1-6 hepatocytes incubated with or without insulin or miR-29b mimics. $70 \times 59 \mathrm{~mm}(300 \times 300 \mathrm{DPI})$ 


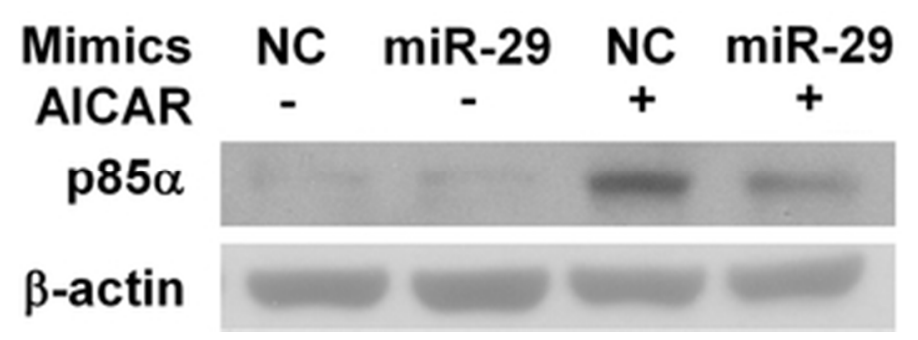

Figure 6 Effects of AICAR and miR-29 mimics on hepatocytes expression of p85a. Expression levels of p85a in hepatocytes incubated with or without miR-29b mimics and AICAR. $28 \times 10 \mathrm{~mm}(300 \times 300 \mathrm{DPI})$ 